\title{
Síndrome de Pai: dos nuevos casos con manifestaciones inusuales
} Pai syndrome: Two new cases with unusual manifestations

\author{
Dra. Victoria Huckstadt ${ }^{a}$ Dra. María E. Heis Mendoza ${ }^{a}$ Dra. Angélica Moresco y Dra. María G. Obregon ${ }^{a}$
}

\section{RESUMEN}

El síndrome de Pai se describe como la presencia de 3 anomalías congénitas: fisura de labio medial, pólipos cutáneos nasales y faciales, y lipoma pericallosal. La expresión clínica es variable. El desarrollo neuromadurativo suele ser normal. Existen 42 casos descritos en la literatura. Se proponen distintos tipos deherencia, pero, hasta la actualidad, no existe un gen asignado para esta patología. Se presentan dos pacientes con síndrome de Pai, uno de ellos con hallazgos clínicos aún no descritos (defectos de segmentación vertebral y osteoma coroideo).

Palabras clave: síndrome de Pai, pólipos nasales, lipoma pericallosal, cuerpo calloso, anomalías vertebrales.

\begin{abstract}
Pai syndrome is a very rare congenital disorder characterized by medial cleft lip, nasal and facial cutaneous polyps, and pericallosal lipoma. Broad phenotypic variability exists in this condition. Neurodevelopment is usually normal. Up to date 42 cases have been reported in the literature. Different types of inheritance have been proposed, but most cases are sporadic. No gene has been identified. We report two cases with Pai syndrome, one of them with novel clinical findings as vertebral segmentation defects and choroidal osteoma.

Key words: Pai syndrome, nasal polyps, pericallosal lipoma, corpus
\end{abstract} callosum, vertebral anomalies.

http:/ / dx.doi.org/10.5546/ aap.2018.e336

Cómo citar: Huckstadt V, Heis Mendoza ME, Moresco A, et al. Síndrome de Pai: dos nuevos casos con manifestaciones inusuales. Arch Argent Pediatr 2018;116(2):e336-e340.

\section{INTRODUCCIÓN}

El síndrome de Pai [SP] (MIM155145) fue descrito por G. S. Pai como la presencia de 3 anomalías congénitas: fisura de labio medial, pólipos cutáneos nasales y faciales, y lipoma

a. Servicio de Genética, Hospital de Pediatría Garrahan, Ciudad Autónoma de Buenos Aires, Argentina.

Correspondencia:

Dra. Victoria Huckstadt: vickyhuckstadt@gmail.com.

Financiamiento: Ninguno.

Conflicto de intereses: Ninguno que declarar.

Recibido: 28-8-2017

Aceptado: 23-10-2017 pericallosal en el sistema nervioso central [SNC]. ${ }^{1}$ La expresión clínica es variable, ya que puede presentar anomalías oculares, nariz bífida, úvula bífida, diastema dentario y alopecia frontal. El neurodesarrollo suele ser normal ${ }^{2-5}$ y se han reportado convulsiones en pacientes con lipoma pericallosal. ${ }^{4,6,7}$ Existen 42 casos descritos en la literatura, con una incidencia estimada en 1 de cada 20 000-40 000 recién nacidos. ${ }^{6,8}$

La mayoría de los casos comunicados de SP son esporádicos y se proponen distintos tipos de herencia e, incluso, algunos autores proponen la etiología multifactorial. ${ }^{9-11}$ Masuno y colaboradores describen a un paciente con translocación aparentemente balanceada entre los brazos largos de los cromosomas X y 16 (cariotipo: 46, X, t(X; 16) (q28; q11.2)), y plantearon que esta podría ser la región implicada, ${ }^{12}$ pero, hasta la actualidad, no se han reportado otros pacientes con anomalías cromosómicas y no existe un gen asignado para esta patología.

Se presenta a dos pacientes con SP evaluados en el Servicio de Genética, uno de ellos con hallazgos clínicos aún no descritos.

\section{CASOS CLÍNICOS \\ Paciente 1}

Paciente de sexo femenino, derivada al Servicio de Genética a los 9 meses de vida por presentar un pólipo nasal y mamelones preauriculares. Era la única hija de padres sanos, no consanguíneos, nativos argentinos. Madre de 33 años y padre de 34 años. Embarazo complicado con diabetes gestacional. Nació por cesárea a las 37 semanas, pesó 3630 g (p 90), con perímetro cefálico de $36 \mathrm{~cm}$ (p 97), talla de $48 \mathrm{~cm}$ (p 50), Apgar 8/9. Al momento de la consulta, presentaba desarrollo neuromadurativo acorde a la edad, con peso de $8340 \mathrm{~g}$ ( $\mathrm{p} 25-50)$, talla de $68,5 \mathrm{~cm}$ ( p 10-25), perímetro cefálico de $45,6 \mathrm{~cm}$ (p 75), asimetría facial, frente hirsuta, sinofris, telecanto, dermoide epibulbar en el ojo izquierdo. Pólipo nasal sésil en columela, que obstruía la fosa nasal izquierda, fosita sobre filtrum. Paladar y úvula completos. Fisura labial lateral transversa izquierda pequeña. Mamelones preauriculares bilaterales, orejas en copa con hélices plegados. 
Cuello corto (Figura 1 A y B).

A los 14 meses de vida, se le realizó exéresis quirúrgica del pólipo nasal y mamelones preauriculares, cuya anatomía patológica informó un tejido fibroepitelial.

Se detectó, en los estudios complementarios, hipoacusia neurosensorial profunda bilateral (actualmente, equipada con audífonos); lipoma supracalloso con calcificaciones e hipoplasia de cuerpo calloso en la tomografía computada [TC] del SNC y, en la columna cervical, múltiples anomalías óseas de cuerpos vertebrales por alteraciones en la fusión-segmentación de arcos posteriores (Figura 2); radiografía de columna con defectos de segmentación vertebral a nivel cervical y dorsal. A los 2 años de vida, se le diagnosticó un osteoma coroideo en el ojo derecho, y se realizó un tratamiento quirúrgico por el Servicio de Oftalmología. El ecocardiograma, la ecografía abdominal y renal fueron normales.

\section{Paciente 2}

Paciente de sexo femenino, derivada al Servicio de Genética a los 2 meses de vida por presentar un pólipo nasal y mamelones preauriculares. Era la segunda hija de padres sanos, no consanguíneos, oriundos de Perú, de 23 años la madre y 27 años el padre. Hermana mayor, sana. Embarazo sin complicaciones. Nació de 39 semanas por parto vaginal, $3170 \mathrm{~g}$ (p 50), Apgar 9/10. Fue seguida hasta los 4 años de vida y presentó desarrollo neuromadurativo normal, peso de $4,4 \mathrm{~kg}$ (p 50), talla de $53 \mathrm{~cm}(\mathrm{p} 3)$, perímetro cefálico de $36,5 \mathrm{~cm}$ (p 10), telecanto, orejas en copa, de implantación baja y rotación posterior con mamelones bilaterales por delante del trago. Narinas hipoplásicas, la derecha presentaba un pólipo pediculado (Figura $3 \mathrm{D}$ y E).

Al año de vida, se realizó la exéresis del pólipo nasal y los mamelones preauriculares, cuyo informe anatomopatológico refirió tejido cutáneo y adiposo.

Se detectó, en los estudios complementarios, la comunicación interventricular [CIV] subtricuspídea en el ecocardiograma, la cual era asintomática y no requirió tratamiento, coloboma inferior de la retina en el ojo izquierdo en el fondo de ojo, TC de macizo facial con un lipoma subcutáneo en ala nasal derecha e intracoanal. Las otoemisiones acústicas, la ecografía abdominorrenal, la TC cerebral y la radiografía de columna eran normales.

FIgURA 2. Tomografía computada cervical del paciente 1, defecto de la segmentación vertebral

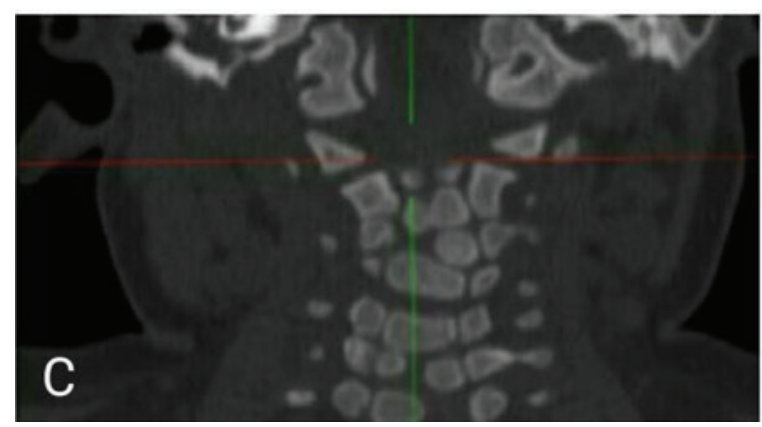

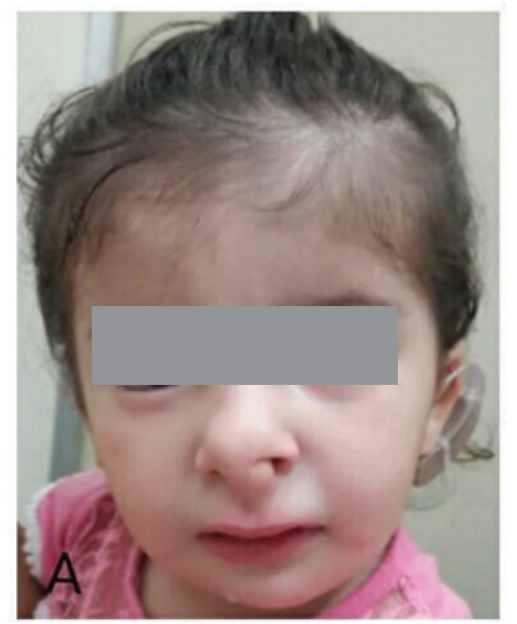

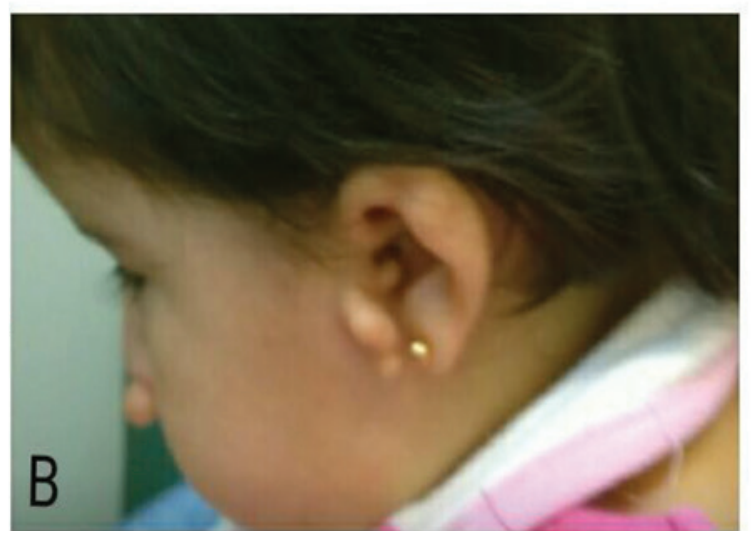




\section{DISCUSIÓN}

El SP es un trastorno congénito muy poco frecuente. Considerando los pacientes aquí descritos, existen 44 casos reportados hasta la actualidad. Las características clínicas de nuestros pacientes y los de la bibliografía se detallan en la Tabla 1.

Según los trabajos revisados, es mayor la frecuencia de esta entidad en mujeres que en varones (con una relación de 1:0,5).

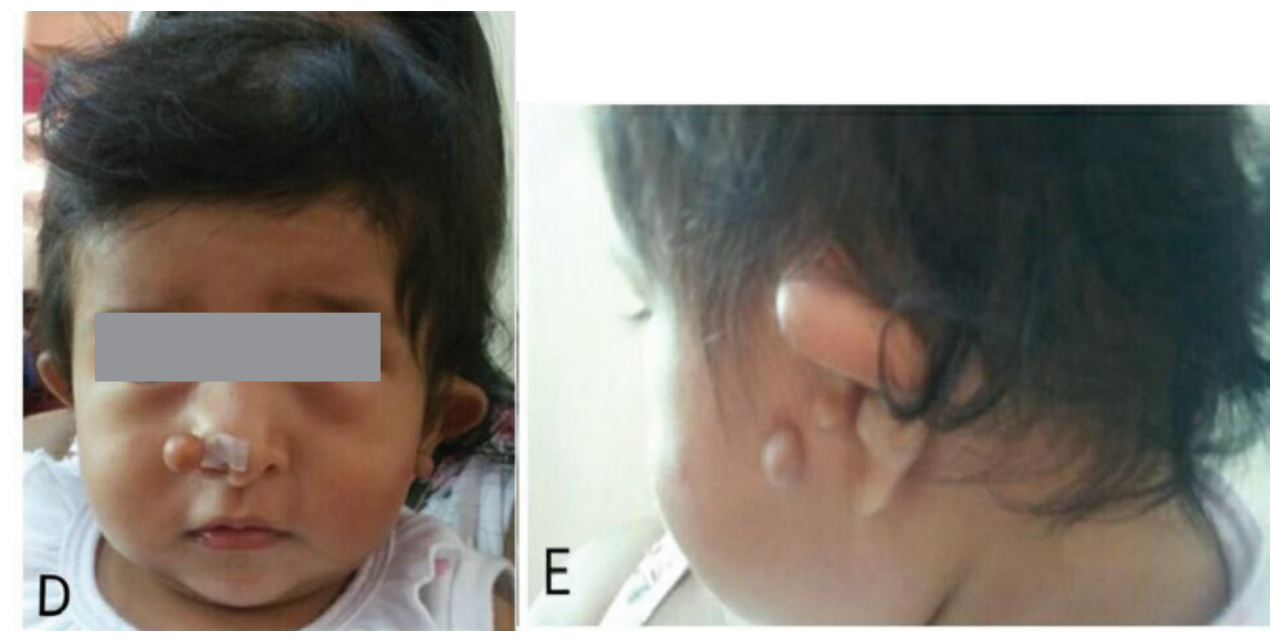

Tabla 1. Hallazgos clínicos de pacientes con síndrome de Pai

\begin{tabular}{lcccc}
\hline & Casos reportados previamente $\left(^{*}\right)$ & Paciente 1 & Paciente 2 & Total \\
\hline $\mathrm{N}$ & 42 & 1 & 1 & 44 \\
Sexo & $26 \mathrm{~F}: 16 \mathrm{M}$ & $\mathrm{F}$ & $\mathrm{F}$ & $28 \mathrm{~F}: 16 \mathrm{M}$ \\
Pólipo facial & 23 & - & - & $23(52,27 \%)$ \\
Pólipo nasal & 41 & + & + & $43(97,72 \%)$ \\
Fisura de labio y/o paladar & 29 & + & - & $30(68,18 \%)$ \\
Frénula maxilar & 9 & - & - & $9(20,45 \%)$ \\
Fosita facial y/o paladar & 8 & + & - & $9(20,45 \%)$ \\
Lipoma pericalloso & 37 & + & - & $38(86,36 \%)$ \\
Hipoplasia/agenesia de cuerpo calloso & 13 & + & - & $14(31,81 \%)$ \\
Anomalía ocular & 12 & + & + & $14(31,81 \%)$ \\
Mamelón preauricular & 3 & - & $5(11,36 \%)$ \\
Implantación anómala del cabello & 16 & + & - & $16(36,36 \%)$ \\
Defecto vertebral & 0 & - & - & $1(2,27 \%)$ \\
Cardiopatía & 1 & + & $2(4,54 \%)$ \\
\hline
\end{tabular}

(*) Pai (1987), Reardon (1990), Rudnik (1994), Masuno (1997), Mishima (1999), Al-Mazrou (2001), Da Silva (2003), Coban (2003), Szeto (2005), Lees (2006), Castori (2007), Guion-Almeida (2007), Chousta (2008), Vaccarella (2008), Savasta (2008), Guion-Almeida Richieri-Costa (2009), Ochoa (2010), Andeeva (2010), Zanetta (2011), Lederer (2012), Abdelmaaboud (2012), Arconada (2013), Blouet (2014), Dobrocky (2014), Azurdia (2014), Abdollahifakhim (2014), Mae Hong (2014), Melloni (2015), Martins (2015), Ferreira (2016), Caycedo (2016), Tormei (2017), Surenda (2017).

N: número de pacientes. F: femenino. M: masculino. (+) Presencia. (-) Ausencia. 
El pólipo nasal congénito es un signo relevante en el SP, ya que está presente en nuestros pacientes y en 41 de los 42 casos revisados $(97,72 \%)$. Los que fueron biopsiados se describen histológicamente como tejido dermoide o hamartomatoso. ${ }^{4,9,12}$ La fisura de labio o paladar se encontró en el $68,18 \%$ de los pacientes.

El lipoma pericallosal, presente en el paciente 1, se encuentra en el $86,36 \%$ de los casos de la bibliografía; es una lesión rara y está frecuentemente asociada con otras lesiones intracerebrales, como malformación del cuerpo calloso (31,81\% de los casos revisados). ${ }^{1,2,5,13}$ Nuestros pacientes presentan mamelones preauriculares, y el paciente 1, fosita en filtrum, características ya comunicadas en la literatura. ${ }^{4,5,14}$ Nuestra paciente 2 es el segundo caso con CIV. ${ }^{12}$ Aunque está ampliamente descrita la afectación ocular en el SP, ${ }^{6,14,15}$ (31,81\% de los casos revisados), el osteoma coroideo (tumor benigno de la coroides muy raro, capaz de producir tejido óseo en coroides y afectación visual grave) no ha sido informado previamente.

Desde su primera descripción por G. S. Pai, se ha planteado como diagnóstico diferencial de este síndrome la displasia frontonasal (MIM 136760, 316451 y 613456) por la gran superposición clínica entre ambas entidades ${ }^{1,2,4,5,13}$ e, incluso, se plantea que formarían parte del mismo espectro. La presencia de defectos de segmentación vertebral de nuestra paciente (que no ha sido comunicada hasta la actualidad en el SP) y la cardiopatía congénita obligan a plantear como otros diagnósticos diferenciales el espectro oculoauriculovertebral (MIM 164210) y el síndrome de Wildervanck (MIM 314600). Pero no hay casos comunicados hasta el momento de estas entidades con pólipos nasales.

Hasta la actualidad, la etiopatogenia es desconocida. Cuarenta de los casos revisados son de presentación esporádica. Algunos autores plantean una herencia autosómica dominante basada en que Rudnik y colaboradores comunican un paciente de sexo masculino cuyo padre presenta un coloboma de iris, ${ }^{10}$ y Lees y colaboradores reportan la presencia de dermoide nasal junto con la fisura del labio medial en 5 generaciones. ${ }^{11}$ Al-Mazrou y colaboradores proponen, incluso, una etiología multifactorial por la discordancia en gemelos monocigóticos. ${ }^{9}$ Fueron estudiados 16 de los 42 pacientes de la literatura con cariotipo y dos de ellos con array de hibridación genómica comparativa (comparative genomic hybridization array; CGH-array, por sus siglas en inglés), con resultados normales, salvo en el caso de Masuno y colaboradores, en el que se halló una translocación aparentemente balanceada (46, X, t (X; 16) (q28; q11.2)) en una paciente que, además de las características de SP, presentaba retraso neuromadurativo y baja talla. ${ }^{12}$

Los defectos del desarrollo de la línea media, como los encontrados en el SP, son inducidos en etapas tempranas de la embriogénesis. La fisura de labio o paladar medial es una anomalía poco frecuente, resultado de la fusión incompleta de los procesos nasales mediales por migración fallida del mesodermo en los días 19-21 de gestación. Esta anomalía, muchas veces, está asociada a malformaciones intracerebrales con pérdida variable de estructuras de la línea media. El cuerpo calloso se desarrolla posteriormente en la semana 10; puede mostrar una afectación variable, que va desde ligeras anomalías hasta agenesia completa. Los lipomas del SNC se originan por la proliferación de las células grasas presentes en las leptomeninges, una estructura embriológica normalmente diferenciada de las meninges primitivas. ${ }^{13}$

\section{CONCLUSIÓN}

El SP en una entidad poco conocida. Aunque hay pocos casos reportados hasta la actualidad, se cree que la incidencia está subestimada. La comunicación de nuevos casos de este síndrome puede resultar útil en trabajos futuros que intenten ampliar el entendimiento de su etiopatogenia para un correcto asesoramiento genético.

Es importante que el pediatra, ante la presencia de pólipos nasales congénitos, sospeche esta entidad y oriente los estudios complementarios con un enfoque multidisciplinario para detectar anomalías asociadas, como, por ejemplo, lipoma pericallosal, que es frecuente en esta entidad.

\section{REFERENCIAS}

1. PaiGS, LevkoffAH, Leithiser REJr. Median cleft of the upper lip associated with lipomas of the central nervous system and cutaneous polyps. Am J Med Genet 1987:26(4):921-4.

2. Guion-Almeida ML, Mellado C, Beltrán C, et al. Pai syndrome: report of seven South American patients. Am J Med Genet A 2007:143A(24):3273-9.

3. Guion-Almeida ML, Richieri-Costa A. Severe midline craniofacial anomalies: Overlap with Pai syndrome. Clin Dysmorphol 2009;18(3):154-7.

4. Lederer D, Wilson B, Lefesvre P, et al. Atypical findings in three patients with Pai syndrome and literature review. Am J Med Genet A 2012;158(11):2899-904.

5. Castori M, Rinaldi R, Bianchi A, et al. Pai syndrome: first patient with agenesis of the corpus callosum and 
literature review. Birth Defects Res A Clin Mol Teratol 2007;79(10):673-9.

6. Zanetta A, Cuestas G, Oviedo M, et al. Obstrucción nasal unilateral en niños. Síndrome de Pai. Arch Argent Pediatr 2011;109(5):100-3.

7. Martins A, Cruz D, Fitz Maurice MA, et al. Síndrome de Pai. Rev Pediatr Elizalde 2015;6(1-2):1-54.

8. Melloni-Magnelli L, de La Garza-Giacoman R, MartínezLeija H, et al. Primer caso de Síndrome de Pai en México. Cir Plást Iberolatinoam 2015;41(2):183-9.

9. Al-Mazrou KA, Al-Rekabi A, Alorainy IA, et al. Pai syndrome: a report of a case and review of the literature. Int J Pediatr Otorhinolaryngol 2001;61(2):149-53.

10. Rudnik-Schoneborn S, Zerres K. A further patient with Pai syndrome with autosomal dominant inheritance? J Med Genet 1994;31(6):497-8.
11. Lees MM, Connelly F, Kangesu L, et al. Midline cleft lip and nasal dermoids over five generations: a distinct entity or autosomal dominant Pai syndrome? Clin Dysmorphol 2006;15(3):155-9.

12. Masuno M, Imaizumi K, Fukushima Y, et al. Median cleft of the upper lip and pedunculated skin masses associated with de novo reciprocal translocation $46, X \mathrm{t}(X ; 16)$ (q28; q11.2). J Med Genet 1997;34(11):952-4.

13. Vaccarella F, Pini Prato A, Fasciolo A, et al. Phenotypic variability of Pai syndrome: report of two patients and review of the literature. Int J Oral Maxillofac Surg 2008;37(11):1059-64.

14. Abdelmaaboud M, Nimeri N. Pai syndrome: first reported case in Qatar and review of literature of previously published cases. BMJ Case Rep 2012;2012:bcr0220125940.

15. Tormey P, Bilic Cace I, Boyle MA. Ocular dermoid in Pai Syndrome: A review. Eur J Med Genet 2017;60(4):217-9. 